\title{
CCDM: Um Modelo de Terceirização em Laboratório de Prestação de Serviços
}

\author{
Nelson Guedes de Alcântara \\ Sebastião Elias Kuri
}

\author{
Universidade Federal de São Carlos - UFSCar \\ Departamento de Engenharia de Materiais - DEMa \\ Centro de Caracterização e Desenvolvimento de Materiais - CCDM \\ Via Washington Luiz, km 235 - São Carlos-SP 13565-905 \\ Tel.: 016-2720707 - Fax.: 016-2720160 \\ E-mail: nelsong@power.ufscar.br
}

Palavras-chaves: Gestão de Pesquisa e Desenvolvimento, Interação UniversidadeEmpresa, Centro de P\&D, Terceirização.

Key words: R\&D Management, Outsourcing, Services, University-Industry Relationship

\section{RESUMO}

$O$ aumento nos custos de equipamentos de Pesquisa e Desenvolvimento - P\&D além da necessidade de aperfeiçoamento constante da mäo-de-obra especializada, tem sido uma das principais razōes para o fechamento de vários laboratórios de P\&D das indústrias. Entretanto, como a pesquisa e desenvolvimento sāo essenciais para novos produtos em um mencado cada vez mais competitivo, diversos centros de P\&D foram criados nos países desenvolvidos. No Brasil, o primeiro centro de prestação de serviços criado pelo Estado está localizado no campus da Universidade Federal de Sāo Carlos: o Centro de Caracterização e Desenvolvimento de Materiais - CCDM, um projeto de US\$ 5 milhōes. A principal diferença entre o CCDM e os existentes centros de P\&D no Brasil, é a de que o CCDM é uma entidade sem fins lucrativos, deve ser auto-sustentável e presta serviços para as empresas, universidades e institutos de pesquisas. Este trabalho apresentará a experiência deste primeiro centro no Brasil. O crescimento contínuo e o sucesso deste centro - exemplo de terceirização - deve conduzir a um progresso na pesquisa e desenvolvimento na área de Ciência e Engenharia de Materiais.

\section{ABSTRACT}

The increase of reseanch and development equipment costs and the need of updating specialized staff has been the main reason for the cease of several industrial R\&D laboratories. As R\&D is essential for new products in a very competitive market, several industry-university cooperative reseanch centers have been created in the developed countries. In Brazil the first center created by the government is located at the Federal University of Säo Carlas: the Center for Characterization and Development of Materials - CCDM, a US\$ 5 millions project. The main difference among CCDM and the existing R\&D centers in Brazil is that the CCDM is a non-profitable institution, has to be selfsupported and provides services to the industries, universities and research centers. This paper will present the experience of this first center in Brazil. The continued growth and success of this outsourcing center should lead to considerable progress in reseanch and development in the field of Materials Science and Engineering. 


\section{Introdução}

O Centro de Caracterização e Desenvolvimento de Materiais - CCDM, criado pela Universidade Federal de São Carlos - UFSCar, com participação da Universidade Estadual Paulista "Júlio de Mesquita Filho" - UNESP, é uma organização que tem por finalidade a prestação de serviços de apoio à pesquisa $\mathrm{e}$ ao desenvolvimento científico e tecnológico na área de Ciência e Engenharia de Materiais - CEM.

Implantado com recursos do Ministério da Ciência e Tecnologia - MCT através do Programa de Apoio ao Desenvolvimento Científico e Tecnológico - PADCT e gestão financeira da Financiadora de Estudos e Projetos - FINEP, com empréstimo junto ao Banco Mundial - BIRD, o centro também conta com algumas contrapartidas institucionais da UFSCar e UNESP, e apoio do Programa de Capacitação de Recursos Humanos para o Desenvolvimento Tecnológico - RHAE e Conselho Nacional de Desenvolvimento Científico e Tecnológico - CNPq, para cumprir seus objetivos.

Contando com equipamentos de última geração e recursos humanos especializados e em constante atualização, além de um quadro de assessores de reconhecida excelência em Materiais, o CCDM atua em parceria com indústrias, universidades $\mathrm{e}$ institutos de pesquisa que necessitam de ensaios, consultoria, treinamento especializado, pesquisa, desenvolvimento científico e tecnológico, projetos integrados e atualização tecnológica em caracterização de materiais cerâmicos, metálicos, poliméricos e compósitos.

O presente trabalho apresentará a importância desta nova concepçāo de laboratório de prestação de serviços no Brasil - modelo de terceirização - cujo crescimento contínuo e sucesso deve conduzir a um progresso na pesquisa $e$ desenvolvimento na área de Ciência e Engenharia de Materiais.

\section{A Concepção}

Existe um razoável consenso da importância do desenvolvimento científico e tecnológico no processo de geração de riquezas e aprimoramento social de um país. Mesmo reconhecendo que o Brasil não poderá alcançar a curto e médio prazo o desenvolvimento científico-tecnológico dos paises líderes em ciência e tecnologia EUA, Japão, Alemanha - verifica-se que para a maioria dos países as diversas áreas do conhecimento que são passíveis de influência do avanço tecnológico são: novos materiais, biotecnologia e engenharia genética, informática e informaçōes, química fina e ecologia ${ }^{(1)}, \mathrm{e}$ portanto são estratégicas.

$\mathrm{Na}$ indústria, a maior parte da tecnologia aplicada depende em muito dos materiais convencionais e dos novos materiais. Em países em desenvolvimento como o Brasil, onde existe a incomoda dependência científica e tecnológica, podese observar enormes dificuldades em desenvolver tecnologia própria, principalmente por possuir pouco conbecimento de como desenvolver e produzir os materiais adequados. É inadmissível que o país, embora possuidor 
de enormes reservas nativas, seja exportador de matérias primas para posterior importação de produtos manufaturados com alto valor tecnológico $\mathrm{e}$ custo agregado.

Nos últimos anos o governo brasileiro através de suas agências de fomento à pesquisa investiu numa política de capacitação tecnológica para a indústria, visando incentivá-las ao desenvolvimento $\mathrm{e}$ as inovaçōes tecnológicas, seleção, absorção, adaptação, aperfeiçoamento e difusão das tecnologias nacionais $e$ importadas. Este apoio a capacitação tecnológica da indústria, está sendo desenvolvido com ênfase na formação e desenvolvimento de recursos humanos, $\mathrm{e}$ na adequação da infra-estrutura tecnológica, compreendendo a modernização dos institutos tecnológicos, laboratórios, centros de pesquisas de universidades, e a criação de novos centros em áreas emergentes ${ }^{(1)}$, como é o caso de Materiais.

Partindo do pressuposto de que a Ciência e Tecnologia - C\&T constituem o fundamento moderno do desenvolvimento econômico (1), o governo brasileiro através do MCT estabeleceu dentro do PADCT, a criação de um centro de prestação de serviços na área de Caracterização de Materiais visando atender a comunidade industrial e acadêmica.

Este centro deveria ter uma estrutura centralizada própria e procuraria atender a todas as solicitações em caracterização e desenvolvimento de materiais; entretanto, é bom salientar, que é improvável que uma única estrutura possa atender à todas as necessidades de desenvolvimento e caracterização de materiais do Brasil.

A origem desta concepção foi baseada na experiência existente nos EUA, quando a partir da década de 70 foram criados 53 centros de Pesquisa e Desenvolvimento P\&D em diversas áreas do conhecimento científico e tecnológico, e que deveriam relacionar-se diretamente com o setor industrial. Estes centros de P\&D situam-se principalmente dentro das universidades, $\mathrm{e}$ são basicamente sustentados com recursos provenientes de empresas associadas ou clientes, bem como de recursos a fundo perdido oriundo de agências governamentais de fomento científico $e$ tecnológico (2).

Aqui no Brasil o primeiro centro com estas características, o Centro de Caracterização e Desenvolvimento de Materiais, foi instalado numa universidade pública federal, comprometida historicamente com o desenvolvimento científico e tecnológico do país. O edital para a sua implantação ocorreu em 1991 sendo vencedora a proposta apresentada pela UFSCar e UNESP, devido principalmente a grande interação já existente entre o Departamento de Engenharia de Materiais - DEMa da UFSCar e o setor industrial, e pela grande qualificação do pessoal envolvido - cerca de 70 doutores - atuando na área de CEM. Desta forma foi implantado o CCDM no campus da UFSCar, em São Carlos.

Nas universidades públicas brasileiras tem-se observado uma redução gradativa nos recursos para as pesquisas, $e$, muitas vezes, mesmo tendo pessoal capacitado e equipamentos disponíveis, nāo há recursos suficiente para a sua manutenção. Por 
outro lado, o setor empresarial tem maiores facilidades para disponibilizar recursos para projetos de pesquisa e desenvolvimento de seu interesse, porém, é extremamente onerado na manutenção da infraestrutura de equipamentos e pessoal qualificado de um centro de $P \& D$, necessário para o sucesso de suas açōes de natureza científica e tecnológica. $O$ centro visa complementar a interação entre as necessidades do setor industrial e a capacitação existentes nas universidades, pois possui infraestrutura e pessoal especializado, e tem como atividade principal a prestação de serviços.

Tanto o setor universitá rio como o empresarial podem se beneficiar da estrutura do centro. Do ponto de vista acadêmico, um maior número de pesquisadores poderá ter acesso aos equipamentos de última geração que são necessários para as suas atividades de pesquisa, economizando-se recursos do Estado que seriam utilizados na eventual duplicação dos equipamentos de pesquisa. No que se refere ao setor empresarial o centro representa uma efetiva possibilidade de apoio para a solução técnica de seus problemas industriais, reduzindo os custos de investimentos e de manutenção de uma estrutura própria de P\&D. Dado que o centro é uma entidade sem fins lucrativos mas que tem como objetivo alcançar a sua auto- sustentação, estabeleceu-se uma política coerente de definiçāo dos custos de seus serviços, tanto para o setor acadêmico quanto para o empresarial, que será responsável pela composição do seu orçamento anual.

Alguns outros aspectos também podem ser observados na concepção do CCDM. Os recursos iniciais foram da ordem de US\$ 5 milhões, enquanto que nos EUA os investimentos foram no mínimo 4 vezes superiores. Estes recursos iniciais para o centro no Brasil foram exclusivamente para sua implantação, enquanto que nos Estados Unidos houve além dos recursos de implantação aporte financeiro decrescente em um período variando de 5 a 10 anos para manutenção da estrutura até sua autosustentação. Sabendo-se que nos EUA há uma grande tradição científica $e$ tecnológica, e que normalmente as empresas interagem com as instituições de pesquisa das universidades, pode-se verificar uma falha na concepção do primeiro centro auto-sustentável do Brasil, pela ainda pequena, apesar de crescente, sintonia entre as universidades e o setor industrial, mas principalmente pela dificuldade em se difundir ou vender C\&T no país. Dado que a implantação da infraestrutura física do CCDM foi concluída com sua inauguraçāo em 16 de março de 1995, e que os recursos governamentais para tal terminaram em julho de 1995, serão abordadas as várias estratégias que estāo sendo utilizadas para sua manutençāo e as açôes que estāo sendo tomadas para atingir a auto- sustentação, preservando a qualidade e eficiência de seus serviços.

\section{A Implantação}

Para a seleção dos equipamentos já comprados, instalados e em pleno funcionamento, foram reunidos três grupos de professores doutores em CEM, a se 
saber: em Caracterização Química, Caracterização Física e Análise Microestrutural de Materiais. Foram escolhidos principalmente equipamentos analíticos que pudessem atender a maior diversificação possível de materiais, já que deveria $m$ atender as necessidades das indústrias, das universidades e de outros centros de pesquisas. O levantamento inicial indicou que seriam necessários cerca de US $\$ 20$ milhōes para atender a demanda na área, entretanto, dado a limitação dos valores existentes no edital, foi realizada a proposta com a inclusão de cerca de US\$ 4 milhões em equipamentos.

Para a operacionalização financeira, utilizaram-se os mecanismos administrativos da Fundação de Apoio ao Desenvolvimento Científico e Tecnológico - FAI.UFSCar, uma entidade de direito privado mas vinculada aos interesses da UFSCar. Tal decisão foi correta, já que foi possível realizar a implantaçāo da infraestrutura $e$ a compra dos equipamentos dentro de tempos recordes, atendendo à todos requisitos legais de licitação, inclusive internacional. Para se ter uma idéia de como esta agilização foi importante, havia uma previsão de gastos de até $20 \%$ para taxas de importação dos equipamentos, ou seja cerca de US\$ 800 mil, entretanto a média dos custos reais ficaram em torno de $8 \%$, ou seja em torno de US\$ 320 mil. Estes recursos que eram para ter sido gastos em taxas de importação foram aplicados na aquisiçāo de um número bem maior de assessórios para os equipamentos, ampliando assim as facilidades existentes.

Outro fator importante a ser considerado foi o cuidado na realizaçāo das licitações internacionais, onde equipamentos de última geração deveriam possuir no máximo o preço praticado no mercado internacional, e as garantias de manutenção dos equipamentos seriam feitas por representantes locais, o que poderia ser um problema, já que alguns representantes comerciais prometem além das suas condições técnicas para a manutenção. O resultado foi bastante favorável pois cientes de que os equipamentos estariam instalados em um centro de referência nacional e internacional, ou seja, onde haveria um grande número de clientes de empresas, universidades e institutos de pesquisa potenciais compradores destes equipamentos, várias propostas foram feitas com valores inferiores ao do mercado internacional. Por exemplo, um dos equipamentos completo com acessórios tem um preço médio de US\$ 1,1 milhão no país de origem. Dado o interesse da empresa na venda deste equipamento - que em vários casos foram os primeiros a serem instalados no Brasil - a proposta vencedora foi inferior a US $\$ 700 \mathrm{mil}$. Com estas ações o centro tem hoje cerca de $30 \%$ mais de equipamentos e acessórios do que previsto no projeto inicial. Convém acrescentar que as propostas vencedoras incluíram garantia de manutenção dos equipamentos - sem ônus - pelos primeiros dois a nos após a instalação, e treinamento de operação e manutenção do equipamento no Brasil e no pais de origem para os Especialistas do CCDM.

O centro está instalado e em pleno funcionamento em uma área construída de 


\section{PRODUÇĀO}

$1.000 \mathrm{~m}^{2}$. Dado o desenvolvimento de seu Programa da Qualidade, os laboratórios foram adequados segundo normas de segurança para usuários e equipamentos, bem como foi realizado um extenso trabalho de identificação visual.

Os equipamentos existentes são de última geração e possuem precisão adequada à maioria das solicitações das empresas, universidades e institutos de pesquisas. As principais técnicas relacionadas aos equipamentos disponíveis são: Análise Microestrutural: Microscopia Eletrônica de Varredura (SEM) com EDS e WDS, Microscopia Eletrônica Analítica de Tra nsmissão (TEM) com EDS e EELS, Espectromicroscopia de Superfícies com XPS (ESCA), AES, SAM, ISS e XPI, Microscopia Ótica com Análise de Imagens; Caracterização Física: Sistema de Análise Térmica (TGA-DTA-DSC / DSC / Termodilatometria), Difração de Raios-X, Picnometria de Hélio, Análise de Distribuição de Tamanho de Partículas por Atenuação de Raios-X, Análise de Adsorção Física (BET), Porosimetria de Mercúrio; Caracterização Química: Espectrometria de Emissão por Plasma (ICP), Analisador Elementar de C e S, Espectrometria no Infravermelho (FTIR), Espectrometria de Ressonância Magnética Nuclear (NMR), Cromatografia de Permeação em Gel (GPC) e Cromatografia Líquida de Alto Desempenho (HPLC).

Utilizando-se o resultado da experiência dos centros equivalentes nos EUA, colocados em um trabalho que apresentou as causa destes empreendimentos bem sucedidos e dos que fecharam (2), adotou-se que pelo fato do CCDM estar dentro de uma universidade pública brasileira, a primeira comunidade com que se deveria estreitar o relacionamento seria a comunidade universitária interna. Após a constatação de que realmente os princípios e operação deste centro condiziam com os da política universitária, os seus dirigentes, professores pesquisadores, técnicos e administrativos passaram a apoiar cada vez mais o centro. Isto pode ser demonstrado pelo aumento da procura do CCDM por professores e alunos da UFSCar para realizaçāo de seus trabalhos científicos $\mathrm{e}$ tecnológicos. Convém salientar que também esta comunidade acadêmica tem que pagar pelos serviços realizados, já que o centro não recebe recursos da Universidade e deve ser auto-sustentável.

Já em 1995 quando a comunidade universitária interna apoiava cada vez mais as atividades do centro, pôde-se fazer uma divulgação mais forte na comunidade industrial, sendo o resultado bastante favorável, pois 146 empresas e entidades distintas realizaram serviços no centro durante o ano, e a interação universidadeempresa estava realmente acontecendo ${ }^{(3)}$. As empresas vendo a solução de seus problemas, e o centro através de suas facilidades de infraestrutura e do pessoal especializado - interno e de professores da universidade - estava atendendo as empresas com qualidade e dentro dos prazos estabelecidos pelas mesmas.

\section{A Organização}

O CCDM foi estruturado para ser um centro prestador de serviços de excelência, de apoio ao desenvolvimento científico e 
tecnológico na área de Ciência e Engenharia de Materiais, e para tanto estabeleceu-se um planejamento onde foram priorizados sete tópicos principais: $\mathrm{A}$ Estrutura Organizacional, o Sistema da Qualidade, a Motivação, a Capacitação e Atualização dos Recursos Humanos, a Administraçāo das Informações, o Plano Diretor e o Planejamento Estratégico. A partir destes tópicos planejados, houve a sua implantação em uma fase inicial, passando pela capacitação dos $\mathrm{RH}$ e manutenção da estrutura nesta atual fase intermediária, e visando a auto-sustentaçāo e qualidade total de seus serviços a médio e longo prazo.

A estrutura do centro foi estabelecida através de seu Organograma Geral e contempla três Conselhos consultivos e deliberativos, com membros da comunidade acadêmica e empresarial: o Conselho de Administração que define as políticas estratégicas e de ação, o Conselho de Usuários que atua como agente auditor geral, e o Conselho Fiscal, responsável pela auditoria financeira. Já a estrutura funcional contempla uma Diretoria Executiva à qual estão vinculadas a Administração, Assessoria, Sistema da Qualidade, Desenvolvimento Mercadológico e Laboratórios de Caracterização de Materiais.

Ainda dentro desta estrutura estabeleceu-se o Organograma dos Laboratórios de Caracterização de Materiais, possuindo um coordenador geral e três coordenadores de área: Caracterização Física, Caracterização Química e Análise Microestrutural. Também o setor de Desenvolvimento
Mercadológico ficou sob responsabilidade de um coordenador específico.

Partindo da premissa de que o CCDM é um centro prestador de serviços com alto valor agregado científico e tecnológico, estabeleceu-se a prioridade na contratação de mão de obra qualificada, com experiência industrial, e que pudesse atuar na concepção dos serviços tendo como prática o "faça uma vez só, e com qualidade". O quadro de recursos humanos diretamente vinculados ao centro em tempo integral é constituído de 2 pessoas no setor administrativo e 14 técnico-científico - 5 com doutorado, 5 com mestrado e 4 graduados - estando todos em permanente capacitação e atualização tanto no pa ís quanto no exterior, já que todos devem ser Especialistas em sua área de atuação, devendo atender a exigente comunidade industrial e acadêmica.

À dez especialistas técnico-científicos são delegadas responsabilidades pelos equipamentos e técnicas associadas, e para tanto receberam ou estão recebendo treinamento em suas respectivas áreas técnico-científicas além da administrativa, de tal forma que possam gerenciar devidamente o seu respectivo Centro de Custo. Este gerenciamento é feito através da execução de ensaios, manutenção, consultoria, treinamento, cursos, credenciamento, contratação de assessoria especializada externa, participação em eventos técnico-científicos além de outros.

De forma a agilizar a organização interna e o atendimento a cliente a infraestrutura laboratorial foi implementada com microcomputadores conectados a uma rede. Como todas as 


\section{PRODUÇĀO}

informações pertinentes ao centro estão residentes no servidor da rede, nenhuma atividade administrativa deve ser feita mais de uma vez. Esta Administração das Informaçōes onganiza e agiliza a operação do centro, e tem o comprometimento de todo seu pessoal, com isto o setor administrativo consegue operar adequadamente com somente uma Secretária Executiva e uma assistente, auxiliadas por estagiários.

A participação de todo pessoal vinculado ao centro em suas decisões gerais, fez com que o comprometimento de todos fosse efetivo. Realizam-se quatro reuniōes mensais com uma hora de duração no máximo cada uma, a saber: duas reuniōes gerais com participação de todos para informaçōes e resoluçōes gerais, uma reuniāo técnico-administrativa para acompanhamento e sugestão de solução para as pendências e problemas existentes, e uma reuniāo para análise crítica do relatório gerencial que contempla todos os resultados do mês antecedente. Estas duas últimas reuniōes tem participação da diretoria e secretaria executiva e dos cinco coordenadores.

\section{Desempenho Operacional}

Dado que o CCDM requisita uma receita anual de US\$ 1 milhão para sua auto sustentaçāo - sendo US $\$ 540$ mil para pagamento de seu pessoal e encargos sociais, US\$ 320 mil para manutençāo dos equipamentos, e US\$140mil para custeio e capital - e a receita ainda nāo é suficiente para sua auto-sustentação, várias alternativas foram ou estão sendo realizadas para tal: convênio com agências governamentais de fomento - RHAE, CNPq, FAPESP, FINEP etc. - acordos com entidades de classe - SEBRAE, ANPEI, Instituto UNIEMP etc. - acordos com empresas, e em estudo para implantação um Modelo Associativo para empresas e entidades que tem interesse no funcionamento e manutenção do centro.

A previsão de receita para o ano de 1995 foi de US\$ $120 \mathrm{mil}$, e pode-se considerar que o desempenho apresentado foi satisfatório já que a receita efetivada foi de US\$160 mil (3). Para este ano de 1996 a previsão de receita é duplicar a de 1995 , ou seja US\$ 250 mil. Dados de janeiro até setembro demonstram uma crescente procura de empresas e pesquisadores dos serviços, e de sua crescente receita. Até setembro de 1996 esta receita foi superior a US $\$ 300$ mil, já superando a previsão a nual.

Outro indicador importante que está sendo utilizado pelo centro é a de novas visitas recebidas, ou seja, em 1995 foram 525 de empresas, 243 das universidades e 41 de institutos de pesquisas, perfazendo um total de 809 novas visitas ${ }^{(3)}$. De janeiro a setembro de 1996 mais de 800 novas visitas já foram realizadas.

Muito trabalbo ainda está por ser realizado para que o CCDM atinja seu objetivo de ser um centro de referência nacional, com uma receita que permita ser o primeiro centro do gênero autosustentável no Brasil. Entretanto, com as excelentes instalações laboratoriais, pessoa] qualificado, e um mercado global e exigente cada vez mais presente no Brasil, a contribuição que o centro pode oferecer 
ao desenvolvimento científico e tecnológico na área de Ciência e Engenharia de Materiais é bastante promissora.

\section{Conclusões}

- O Centro de Caracterização e Desenvolvimento de Materiais - CCDM é o único centro de prestação de serviços - sem fins lucrativos e que deve ser autosustentável - de apoio ao desenvolvimento científico e tecnológico no país, na área de Ciência e Engenha ria de Materiais,

- Três elementos podem ser destacados em sua prática operacional: os equipamentos e técnicas modernas existentes, a qualificação de seu pessoal e especialistas, e a facilidade de acesso por ter como prioridade a prestaçāo de serviços.

- O crescimento contínuo e o sucesso deste centro - exemplo de terceirização deve conduzir a um progresso na pesquisa e desenvolvimento na área de Ciência $\mathrm{e}$ Engenharia de Materiais.

\section{Agradecimentos}

Os autores agradecem ao PADCT, FINEP, RHAE, CNPq, UFSCar e UNESP pelo financiamento e viabilização do CCDM.

Recebido em: 30/11/96

Recebido após modificaçōes: 15/12/97

Aceito em: 04/08/98

Publicado em: 25/03/99

\section{Bibliografia}

(1) BRASIL. Congresso Nacional. CPMI - Causas e dimensões do atraso tecnológico. Brasília, 1992, $185 \mathrm{p}$.

(2) GEISLER, Eliezer; FURINO, Antonio; KIRESUK, Thomas. Factors in the success or failure of industry-university cooperative research centers. Interfaces. EUA, 20(6):99- 109, nov.-dec. 1990.

(3) ALCÂNTARA, Nelson Guedes. Relatório anual 1995 do CCDM. UFSCar/ UNESP, São Carlos, 1996, 34 p. 